\title{
Chemotherapy in Early Breast Cancer: When, How and Which One?
}

\author{
Marcus Schmidt
}

Department of Obstetrics and Gynecology, Johannes Gutenberg University, Mainz, Germany

\section{Keywords \\ Breast cancer · Prognosis · Chemotherapy}

\section{Summary}

The efficacy of chemotherapy depends on the level of risk of the individual patient. Because of this, careful estimation of the risk level is mandatory. In addition to wellestablished clinicopathological factors, validated gene expression signatures might be useful in selected patients if all other criteria are inconclusive for therapeutic decision-making. If indicated, chemotherapy can be used either after surgery (adjuvant) or before surgery (neoadjuvant). Both approaches lead to comparable long-term survival. The neoadjuvant setting offers the additional opportunity for elaborate translational studies to develop and validate predictive biomarkers and to discover mechanisms of resistance to therapy. If possible, chemotherapy regimens should include both anthracyclines and taxanes. Docetaxel should be used every 3 weeks; better tolerability with equivalent efficacy favors the concurrent over the sequential approach. Paclitaxel, on the other hand, should be administered sequentially, either weekly or every 2 weeks. Especially, intense dose-dense sequential chemotherapy with granulocyte colony-stimulating factor support is very effective in high-risk breast cancer patients. In order to decrease toxicities, anthracycline-free regimens or a shortening of the duration of adjuvant chemotherapy are potential options that should be further explored.

\section{When to Use Chemotherapy in Early Breast Cancer?}

It is evident that the magnitude of chemotherapy benefit depends on the level of risk of the individual patient [1]. In order to avoid both over- and undertreatment, it is advisable to select the appropriate treatment strategy on the basis of a careful risk assessment for each individual patient. According to the most recent St. Gallen consensus recommendations, conventional clinicopathological factors arguing for the indi- cation of chemotherapy were histological grade 3 carcinomas, high Ki-67 levels, low hormone receptor status, human epidermal growth factor receptor 2 (HER2) positivity or triplenegative status, and the involvement of more than 3 lymph nodes [2]. In addition to these time-honored clinicopathological factors, a plethora of novel prognostic and predictive factors has emerged over the last decades. To improve the quality of research on biomarkers, a refined system for biomarker study design and evaluation was introduced that incorporates a revised level of evidence (LoE) scale for tumor marker studies, including those using archived specimens (table 1) [3]. Although fully prospective randomized clinical trials to evaluate the medical utility of a prognostic or predictive biomarker are still considered the gold standard, such trials are costly; so, more efficient indirect 'prospective-retrospective' designs using archived specimens might reach LoE I if validated with consistent results.

Since the field of biomarker evaluation for therapy stratification is rapidly evolving, the American Society of Clinical Oncology (ASCO) updated their recommendations for the use of tumor markers in breast cancer [4]. In addition to the estrogen receptor (ER) and HER2, parameters like urokinase plasminogen activator (uPA), plasminogen activator inhibitor 1 (PAI-1), and certain multiparameter gene expression assays like the recurrence score (RS) showed evidence of clinical utility and were recommended for use in practice.

The RS (Oncotype DX ${ }^{\mathrm{TM}}$ ) is a well-known and broadly used assay using formalin-fixed paraffin-embedded (FFPE) tissue. This reverse transcription-polymerase chain reaction (RT-PCR) assay measures the expression of 21 genes in RNA extracted from FFPE samples of tissue from primary breast cancer. This test was developed specifically for patients with ER-positive node-negative breast cancer patients treated with adjuvant tamoxifen [5] and helps to identify patients with little additional benefit from adjuvant chemotherapy [6]. The independent prognostic and predictive significance of the RS was also confirmed in endocrine-responsive node-positive patients receiving anthracycline-based chemotherapy [7]. Again, only patients with high RS derived substantial benefit from chemotherapy.

\section{KARGER}

Fax +497614520714

Information@Karger.com

www.karger.com
(C) 2014 S. Karger GmbH, Freiburg

$1661-3791 / 14 / 0093-0154 \$ 39.50 / 0$

Accessible online at:

www.karger.com/brc
PD Dr. med. Marcus Schmidt

Department of Obstetrics and Gynecology

Johannes Gutenberg University, Medical School

Langenbeckstr. 1, 55131 Mainz, Germany

marcus.schmidt@unimedizin-mainz.de 
More recently, another gene expression assay using FFPE tissue was developed to assess the prognosis of early breast cancer patients treated with endocrine therapy [8]. This multigene EndoPredict ${ }^{\mathrm{TM}}(\mathrm{EP})$ risk score provided additional prognostic information on the risk of distant recurrence of breast cancer patients, independent from conventional clinicopathologic parameters. Combination of EP with tumor size and number of involved lymph nodes (EPclin) led to an even better prognostication. Additionally, EP improved the prognostic classification derived from common clinical guidelines like the National Comprehensive Cancer Center Network, German S3, and St. Gallen guidelines in ER-positive, HER2negative early breast cancer [9].

Using supervised analysis, genes were identified that were differentially expressed in tumors of node-negative and untreated patients who developed a metastasis within 5 years or remained disease free for at least 5 years [10]. The respective classification algorithm outperformed conventional prognostic factors and was confirmed in subsequent retrospective validation studies (MammaPrint ${ }^{\mathrm{TM}}$ ).

Perou et al. [11] described breast cancer subtypes identified after 2-dimensional hierarchical clustering, which they called luminal, basal like, normal like, and ERBB2 like. These intrinsic subtypes differed in their clinical outcome, their preferential site of relapse, and their response to chemotherapy. A 50-gene subtype predictor was developed using microarray and quantitative RT-PCR data (PAM50) [12]. PAM50 is also commercially available and works in FFPE tissue (Prosigna ${ }^{\mathrm{TM}}$ ). These signatures show analytical and clinical validation. However, evidence for these tests is 'prospectiveretrospective' using archival tissue of completed clinical trials, while prospective evidence is still missing (table 2).

Based on these encouraging findings, the St. Gallen consensus conference recently confirmed a classification based on the recognition of intrinsic biological subtypes within the breast cancer spectrum [2]. Even though the original subtype classification was based on gene expression arrays, immunohistochemical determination of ER, progesterone receptor (PR), HER2, and Ki-67 expression is considered as useful by providing a surrogate subtype classification. This classification simplifies the definition of therapy indications, since the subtypes themselves incorporate many of the risk and predictive factors used in previous consensus recommendations. Essentially, the current recommendations advise chemotherapy for triple-negative and for high-risk luminal A, luminal B and HER2-positive breast cancer patients (table 3). Concerning the latter group of HER2-positive patients, it is mandatory to combine chemotherapy with an anti-HER2 therapy.

Table 1. Revised determination of LoEs using elements of tumor marker studies [3]

\begin{tabular}{lll}
\hline LoE & Category & Validation studies available \\
\hline I & A & none required \\
I & B & 1 or more with consistent results \\
II & B & none or inconsistent results \\
II & C & 2 or more with consistent results \\
III & C & none or 1 with consistent results or inconsistent results \\
\hline
\end{tabular}

Table 2. Commercially available molecular tests

\begin{tabular}{|c|c|c|c|c|}
\hline & Mammaprint $^{\mathrm{TM}}$ & Oncotype $\mathrm{DX}^{\mathrm{TM}}$ & EndoPredict ${ }^{\mathrm{TM}}$ & Prosigna $^{\mathrm{TM}}$ \\
\hline Provider & Agendia & Genomic Health & Sividon & NanoString \\
\hline Type of assay & 70 -gene assay & 21-gene RS & 11-gene assay & 50-gene assay \\
\hline Technique & DNA microarrays & qRT-PCR & qRT-PCR & qRT-PCR \\
\hline Central laboratory & yes & yes & no & no \\
\hline Indication and population studied & prognostic, N0-1 & prognostic, N0-1 ER+ & prognostic, N0-1 ER+/HER2- & prognostic, N0-1 \\
\hline Clinical validation & yes & yes & yes & yes \\
\hline $\begin{array}{l}\text { Prospective-retrospective evidence } \\
\text { (\% of recruited patients) }\end{array}$ & $\begin{array}{l}\text { observational feasibility } \\
\text { study (RASTER) }(73 \%)\end{array}$ & $\begin{array}{l}\text { NSABP B-14 }(14 \%) \text {, } \\
\text { NSABP B-20 }(28 \%) \text {, } \\
\text { SWOG } 8814(40 \%) \text {, } \\
\text { ATAC }(30 \%)\end{array}$ & $\begin{array}{l}\text { ABCSG } 6(19 \%), \\
\text { ABCSG } 8(36 \%), \\
\text { GEICAM/9906 (64\%) }\end{array}$ & $\begin{array}{l}\text { MA.12 }(49 \%) \text {, } \\
\text { MA.5 }(66 \%), \\
\text { ABCSG } 8(40 \%) \text {, } \\
\text { ATAC }(16 \%) \text {, } \\
\text { GEICAM/9906 }(66 \%)\end{array}$ \\
\hline Prospective evidence (pending) & MINDACT & $\begin{array}{l}\text { TAILOR }_{x}, \text { RxPONDER, }_{\text {, }} \\
\text { ADAPT }\end{array}$ & & \\
\hline $\mathrm{LoE}_{2009}$ & II & I & I & I \\
\hline AGO recommendation & $+/-$ & $+^{\mathrm{a}}$ & $+^{a}$ & $+1-$ \\
\hline
\end{tabular}

AGO = Arbeitsgemeinschaft für Gynäkologische Onkologie, CTS = category of tumor marker studies, ER = estrogen receptor, FFPE = formalinfixed paraffin-embedded, HER2 = human epidermal growth factor receptor 2 , LoE = level of evidence, qRT-PCR = quantitative reverse transcription-polymerase chain reaction, $\mathrm{RS}=$ recurrence score.

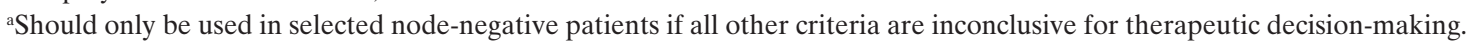


Table 3. Systemic treatment recommendations according to subtypes [2]

\begin{tabular}{ll}
\hline Subtype & Type of therapy \\
\hline Luminal A like & endocrine therapy is the most critical intervention and is often used alone \\
Luminal B like (HER2 negative) & endocrine therapy for all patients, cytotoxic therapy for most \\
Luminal B like (HER2 positive) & cytotoxics + anti-HER2 + endocrine therapy \\
HER2 positive (non-luminal) & cytotoxics + anti-HER2 \\
Triple-negative & cytotoxics \\
\hline
\end{tabular}

Meanwhile, it is becoming increasingly popular to base chemotherapy decisions for patients with luminal disease on genomic results rather than on the surrogate subtype definitions [2]. As outlined above, several multigene molecular assays were recognized as providing accurate and reproducible prognostic information and, in some cases, as providing prediction of response to chemotherapy.

\section{How to Use Chemotherapy in Early Breast Cancer?}

Basically, there are 2 options of how to use chemotherapy: either after surgery (adjuvant) or before surgery (neoadjuvant). It is well accepted that both approaches lead to comparable long-term survival. Beyond local downstaging of large tumors, several advantages speak in favor of investigating new therapies in the neoadjuvant setting. Besides requiring a smaller sample size than adjuvant studies and delivering the efficacy results of a new treatment much earlier, the neoadjuvant setting offers the opportunity for elaborate translational studies to develop and validate predictive biomarkers and discover mechanisms of resistance to therapy. Additionally, interest was raised in whether pathological complete response (pCR) might be a good surrogate for survival. A recent metaanalysis including 11,955 patients from 12 international neoadjuvant trials indeed confirmed that pCR was associated with event-free survival (EFS) and overall survival (OS) [13]. The prognostic value was most pronounced in aggressive tumor subtypes like triple-negative breast cancer (TNBC), HER2-positive or ER-positive poorly differentiated breast cancer. However, at the trial level, there was little association between increases in frequency of pCR and the effect of treatment on EFS or OS. The authors thus concluded that these findings did not support the use of $\mathrm{pCR}$ as a valid surrogate endpoint for improved survival in early breast cancer.

In clinical routine, neoadjuvant chemotherapy could be considered for patients who would otherwise meet the criteria for adjuvant therapy [14]. The largest benefit is expected in those patients with a high likelihood for $\mathrm{pCR}$ (age $<40$ years, tumor size $<2 \mathrm{~cm}$, invasive-ductal histology, poor grade of differentiation, high Ki-67 level, low-level or absent ER, intrinsic subtype basal like or HER2 enriched. In contrast, neoadjuvant treatment should not be recommended routinely when there is uncertainty regarding the indication of chemotherapy. During neoadjuvant treatment, careful monitoring of response is mandatory. Monitoring during treatment must include at least clinical breast examination before each cycle. The frequency of imaging assessment during chemotherapy is a matter of debate. Very recently, a large phase III trial showed that response-guided therapy after only 2 cycles of therapy improved survival especially in ER-positive but not in ER-negative patients [15]. 2,072 patients were treated with 2 cycles of docetaxel, doxorubicin, and cyclophosphamide (TAC) in the neoadjuvant setting. Early responders were randomized to 4 or 6 additional TAC cycles, and early nonresponders to 4 cycles of TAC or vinorelbine and capecitabine (NX) before surgery. Disease-free survival (DFS) was longer in early responders receiving TAC $\times 8$ (hazard ratio $(\mathrm{HR})$ 0.78 ; $95 \%$ confidence interval $(\mathrm{CI}), 0.62-0.97 ; \mathrm{p}=0.026)$ and in early nonresponders receiving TAC-NX than in those receiving TAC $\times 6(\mathrm{HR} 0.59 ; 95 \% \mathrm{CI}, 0.49-0.82 ; \mathrm{p}=0.001)$. DFS was longer after response-guided chemotherapy in all hormone receptor-positive tumors (luminal A HR $=0.55$, luminal B (HER2 negative) HR $=0.40$, and luminal B (HER2 positive) $\mathrm{HR}=0.56)$, but not in hormone receptor-negative tumors (HER2 positive (non-luminal) HR = 1.01 and triplenegative HR = 0.87). This novel response-guided approach could ultimately provide a clinically meaningful advantage for neoadjuvant over adjuvant treatment in early breast cancer [15].

More recently, von Minckwitz et al. [16] examined the efficacy of the addition of carboplatin to neoadjuvant therapy for TNBC and HER2-positive breast cancer. They could show in a randomized phase II study that the addition of neoadjuvant carboplatin to an anthracycline- and taxane-containing regimen significantly increased the proportion of patients achieving a pCR. This improved efficacy was most pronounced in TNBC (odds ratio 1.94; 95\% CI, 1.24-3.04). 53.2\% of TNBC patients allocated to carboplatin showed a pCR as compared to $36.9 \%$ without carboplatin. These results support preclinical data suggesting that TNBC is sensitive to interstrand crosslinking agents. Conversely, the addition of carboplatin to an anthracyclin- and taxane-containing regimen failed to show a higher pCR in HER2-positive breast cancer (32.8\% vs. $36.8 \%$ ). Notably, the addition of carboplatin significantly increased both hematological and non-hematological toxicity. However, these results await confirmation in independent studies.

Considering the choice of chemotherapy, the same regimens should be used for neoadjuvant therapy as in the adjuvant setting. Based on the background outlined above, neoadjuvant chemotherapy is an interesting option that could be 
offered to a substantial proportion of breast cancer patients for whom chemotherapy is indicated.

\section{Which Chemotherapy to Use in Early Breast Cancer?}

The successful history of adjuvant chemotherapy in early breast cancer started almost 4 decades ago when Bonadonna et al. [17] in 1976 published the first report on the efficacy of cyclophosphamide, methotrexate, and fluorouracil (CMF) as adjuvant treatment for node-positive breast cancer.

Later trials showed that substitution of methotrexate with epirubicin (CEF) was even more efficacious both in terms of DFS and OS in premenopausal women with axillary nodepositive breast cancer [18]. The advantage of anthracyclinecontaining 3-drug combinations over CMF was unequivocally confirmed in an individual-patient data meta-analysis of the Early Breast Cancer Trialists' Collaborative Group (EBCTCG) [1]. Altogether, 100,000 patients in 123 randomized trials were included. Anthracycline-based regimens with substantially higher cumulative dosage than standard $4 \times$ AC (adriamycin and cyclophosphamide) (e.g. CAF (cyclophosphamide, adriamycin, and fluorouracil) or CEF) were superior to standard CMF (response rate $(\mathrm{RR}) 0.78 ; \mathrm{p}=0.0004$ ).

\section{Taxanes}

Taxanes received increasing attention since Henderson et al. [19] published a clinical trial in node-positive breast cancer showing that the addition of 4 cycles paclitaxel $175 \mathrm{mg} / \mathrm{m}^{2} \mathrm{im}$ proved DFS (HR 0.83) and OS (HR 0.82). This resulted in an absolute improvement of DFS and OS at 5 years of $5 \%$ and $3 \%$, respectively. However, the design of this trial raised some criticism. On the one hand, the duration of the 2 regimens was strikingly different: 12 weeks for the standard AC compared to 24 weeks for AC followed by paclitaxel. On the other hand, many argued that $4 \times \mathrm{AC}$ was not an optimal standard arm. To circumvent these limitations, the PACS01 trial investigated taxane-containing chemotherapy in early breast cancer which used an adequate anthracycline-containing standard therapy with an identical duration of treatment for comparison [20]. 1,999 node-positive breast cancer patients were randomized to either 6 cycles of FEC (fluorouracil, epirubicin, and cyclophosphamide) every 3 weeks or 3 cycles of FEC followed by 3 cycles of docetaxel every 3 weeks. After a median followup of 60 months, the 5-year DFS and OS rates were $73.2 \%$ and $86.7 \%$ with FEC and $78.4 \%$ and $90.7 \%$ with FECdocetaxel, respectively.

Unlike these trials which used a sequential design, Martín et al. [21] compared a concurrent therapy with TAC (docetaxel $75 \mathrm{mg} / \mathrm{m}^{2}$, doxorubicin (= adriamycin) $50 \mathrm{mg} / \mathrm{m}^{2}$, and cyclophosphamide $600 \mathrm{mg} / \mathrm{m}^{2}$ ) with 5-fluorouracil, doxorubicin, and cyclophosphamide (FAC) as adjuvant chemotherapy for node-positive breast cancer. Long-term follow-up confirmed that the initial therapeutic outcomes seen at the 5-year follow-up with a docetaxel-containing adjuvant regimen were maintained at 10 years [22]. At a median follow-up of 124 months, DFS was $62 \%$ among the 745 patients randomly assigned to receive TAC and 55\% among the 746 patients randomly assigned to receive FAC. The estimated rates of OS at 5 years were $76 \%$ and $69 \%$, respectively. TAC improved DFS relative to FAC irrespective of nodal, hormone receptor, and HER2 status. However, this increased efficacy came with an increased price in the form of toxicities. Shortterm toxicities like febrile neutropenia were markedly increased in patients receiving TAC, which resulted in rates of febrile neutropenia of $24.7 \%$ compared with $2.5 \%$ in patients receiving FAC. The initially high rate of febrile neutropenia after TAC was considerably reduced to $6.5 \%$ when the patients received primary granulocyte colony-stimulating factor (G-CSF) prophylaxis after a protocol amendment [21]. Grade 3-4 heart failure occurred in $26(3 \%)$ patients in the TAC group and $17(2 \%)$ patients in the FAC group, and caused death in 2 patients in the TAC group and 4 patients in the FAC group. A substantial decrease in left ventricular ejection fraction was seen in $58(17 \%)$ patients who received TAC and $41(15 \%)$ patients who received FAC. 6 patients who received TAC developed leukemia or myelodysplasia, as did 3 patients who received FAC [22].

This first generation of taxane-containing trials was conducted almost exclusively in node-positive breast cancer. However, it is evident that node-negative breast cancer patients with adverse prognostic features like poor histological grade, negative ER status, or lymphovascular involvement are also under increased risk of relapse [23]. Accordingly, 1,060 node-negative breast cancer patients with at least 1 additional risk factor were randomized to 6 cycles of FAC or TAC, respectively [24]. At a median follow-up of 77 months, TAC was significantly associated with improved DFS (HR 0.6; 95\% CI, $0.49-0.93 ; \mathrm{p}=0.01)$. This benefit was independent of hormone receptor or menopausal status. Grade 3 or 4 adverse events were significantly more common with TAC (28.2\%) compared with FAC (17.0\%). A comparison of both adjuvant TAC trials clearly shows that the efficacy of TAC is similar in node-positive and node-negative breast cancer patients.

The optimal schedule of (neo)adjuvant chemotherapy (either concurrent or sequential) is still a matter of discussion. Recently, a large study including 3,298 node-positive and HER2-negative breast cancer patients shed some light on this debate. Patients were randomized to either 6 cycles of concurrent TAC every 3 weeks or a sequential schedule of 4 cycles of AC followed by 4 cycles of docetaxel without primary G-CSF prophylaxis [25]. However, despite comparable efficacy across all subgroups, the 2 regimens differed in terms of toxicity. TAC was associated with more febrile neutropenia and thrombocytopenia. Patients randomized to AC followed by docetaxel had more sensory neuropathy $(42.8 \%$ vs. $27.5 \%)$, myalgia ( $50.9 \%$ vs. $35.8 \%$ ), and nail changes $(44.5 \%$ vs. $22.1 \%$ ) as compared to TAC. This differing toxicity profile 
led to a higher rate of discontinuation of therapy in the sequential arm (97 vs. 61 patients). When balancing the comparable efficacy with the differing toxicity profile and duration of treatment of these 2 regimens, the authors concluded that TAC remains an appropriate standard adjuvant chemotherapy regimen for women with early-stage HER2-negative breast cancer.

The aforementioned meta-analysis also included for the first time taxane-based versus non-taxane chemotherapy ( $\mathrm{n}=$ 44,000) [1]. Subgroup analyses of breast cancer mortality showed that taxane-containing therapy slightly but significantly improved the outcome in comparison with an anthracycline-based control regimen (HR 0.872; $\mathrm{p}<0.00001$ ). Proportional risk reductions were little affected by age (up to at least 70 years), nodal status, tumor size, grade of differentiation, ER status, or use of endocrine therapy. Taxane- and anthracycline-based regimens reduced the breast cancer mortality by about one-third. However, the authors clearly stated that this proportional reduction of mortality depended on the level of risk of an individual patient: 'Low absolute risk implies low absolute benefit.'

\section{Dose-Dense Chemotherapy}

In an attempt to increase the efficacy of anthracycline- and taxane-containing regimens in the adjuvant treatment of primary breast cancer, the concept of dose-dense (DD) and sequential chemotherapy received increasing attention. Based on this concept, 2,005 node-positive breast cancer patients were randomly assigned in a $2 \times 2$ factorial design [26]. At a median follow-up of 36 months, DD treatment with G-CSF support improved DFS (HR 0.74; $\mathrm{p}=0.010$ ) as well as OS (HR 0.69; $\mathrm{p}=0.013$ ).

Utilizing an intense DD (IDD) and sequential adjuvant chemotherapy, Moebus et al. [27] conducted a clinical trial in 1,284 high-risk breast cancer patients with 4 or more involved axillary lymph nodes. Patients were randomly assigned to receive IDD sequential epirubicin $\left(150 \mathrm{mg} / \mathrm{m}^{2}\right)$, paclitaxel $(225$ $\left.\mathrm{mg} / \mathrm{m}^{2}\right)$, and cyclophosphamide $\left(2,500 \mathrm{mg} / \mathrm{m}^{2}\right)$ (IDD-ETC) every 2 weeks or conventionally dosed and scheduled epirubicin and cyclophoshamide (EC) followed by paclitaxel every 3 weeks. At a median follow-up of 62 months, IDD-ETC significantly increased the EFS from $62 \%$ to $70 \%$ and OS from $77 \%$ to $82 \%$, respectively. This efficacy was independent of menopausal, hormone receptor, or HER2 status. However, when interpreting these encouraging results, one has to consider that paclitaxel every 3 weeks might not be regarded as an adequate standard anymore.

These 2 trials were included in a meta-analysis investigating DD chemotherapy every 2 weeks with G-CSF support as compared to standard schedules. Altogether 10 studies with 11,989 patients were included in this meta-analysis [28]. In the group with similar dosage and agents in both arms ( 3 trials; 3,337 patients) as well as in the larger group which used different agents and/or dosages in the treatment arms (7 trials;
8,652 patients), DD chemotherapy resulted in significantly better OS (HR 0.84 and 0.85, respectively) and DFS (HR 0.83 and 0.81 , respectively). The rate of non-hematological adverse events was higher in the DD chemotherapy arms than in the conventional chemotherapy arms.

The issue of dose density of paclitaxel or docetaxel was further investigated by the Eastern Cooperative Oncology Group (ECOG) [29]. 4,950 women with axillary lymph nodepositive or high-risk, lymph node-negative breast cancer were enrolled in this trial. After 4 cycles of AC every 3 weeks, patients were randomized to receive either paclitaxel or docetaxel given at 3-week intervals for 4 cycles or at 1-week intervals for 12 cycles. As compared to the control arm (paclitaxel every 3 weeks), the HR for DFS after a median follow-up of 63.8 months was 1.27 among those receiving weekly paclitaxel $\left(80 \mathrm{mg} / \mathrm{m}^{2}\right)(\mathrm{p}=0.006), 1.23$ among those receiving docetaxel every 3 weeks $(p=0.02)$, and 1.09 among those receiving weekly docetaxel $(\mathrm{p}=0.29)$. Weekly paclitaxel was also associated with improved OS (HR 1.32; $p=0.01$ ). The regimens differed in terms of toxicity. There were less grade 3 or 4 adverse events with weekly paclitaxel $(28 \%)$ as compared with $71 \%$ of those receiving docetaxel every 3 weeks $(\mathrm{p}<0.001)$. However, grade 2, 3, or 4 peripheral neuropathy was more pronounced after weekly paclitaxel (27\%).

In an attempt to compare both incorporation of gemcitabine $(\mathrm{G})$ and DD chemotherapy with TAC, Swain et al. [30] randomly assigned 4,894 women with node-positive earlystage breast cancer to 6 cycles of TAC, 4 cycles of DD AC $\rightarrow \mathrm{P}$ ( $\mathrm{P}=$ paclitaxel), or $\mathrm{DD} \mathrm{AC} \rightarrow \mathrm{P}$ with 4 cycles of $\mathrm{G}$ added to the DD paclitaxel (DD AC $\rightarrow$ PG). After 5 years of follow-up there were no significant differences in DFS and OS between these 3 arms.

Conversely, Shulman et al. [31] recently examined in a phase III factorial design the issue of reducing toxicity of DD chemotherapy. They tried to elucidate whether 6 cycles of a chemotherapy regimen were superior to 4 cycles in patients with $0-3$ involved lymph nodes. A total of 3,171 patients were randomly assigned to either 4 or 6 cycles of either AC or paclitaxel. After 2003, all treatment was administered in DD fashion with G-CSF support. The 4-year relapse-free survival (RFS) was $90.9 \%$ and $91.8 \%$ for 6 and 4 cycles, respectively. The HR of 6-4 cycles regarding RFS was 1.03 ( $\mathrm{p}=0.77$ ). The 4-year OS was $95.3 \%$ and $96.3 \%$ for 6 and 4 cycles, respectively, with an HR of $1.12(\mathrm{p}=0.44)$. As expected, toxicity was more severe in the 6-cycle arms. Based on these results, the authors concluded that extending chemotherapy regimens of DD AC or single-agent paclitaxel from 4 to 6 cycles did not improve the clinical outcome but led to increased toxicity. However, shortening adjuvant chemotherapy might be at odds with current guidelines which recommend at least 6 cycles of adjuvant chemotherapy. It might be a reasonable approach to deliver 4 cycles to patients with luminal A breast cancer and large tumor volume or to follow the patient's preference as suggested by Goldhirsch et al. [2]. 
Another way to reduce therapy-induced toxicity is the abandonment of anthracyclines. Jones et al. [32] randomized 1,016 patients with node-positive or high-risk node-negative breast cancer to 4 cycles of either standard-dose AC or docetaxel and cyclophosphamide (TC), administered every 3 weeks. At a median follow-up of 7 years, the difference in DFS between $\mathrm{TC}$ and $\mathrm{AC}$ was significant $(81 \% \mathrm{TC}$ vs. $75 \% \mathrm{AC}$; HR $0.74 ; p=0.033)$ as in OS $(87 \%$ TC vs. $82 \%$ AC; HR 0.69; $\mathrm{p}=0.032$ ). Despite the obvious limitations of a single and rather small clinical trial, the use of anthracyclines in the USA has substantially declined and been replaced by taxane-based regimens [33].

\section{Conclusions}

The efficacy of chemotherapy depends on the level of risk of the individual patient. In addition to well-established clinicopathological factors, validated gene expression signatures might be useful in selected patients if all other criteria are inconclusive for therapeutic decision-making. Besides reducing large tumors in size, neoadjuvant chemotherapy offers an opportunity for 'in vivo' chemosensitivity testing. If not otherwise contraindicated, chemotherapy regimens should include both anthracyclines and taxanes (e.g. TAC or $4 \times \mathrm{AC}$ followed by $12 \times$ paclitaxel weekly). Especially, IDD sequential chemotherapy with G-CSF support is very effective in highrisk breast cancer patients. Since these well-established regimens have slightly differing toxicity profiles, it is mandatory to discuss not only efficacy but also side effects and duration of chemotherapy with the patients.

\section{Disclosure Statement}

M.S. is named inventor on patent applications regarding prediction of chemotherapeutic response in breast cancer and molecular markers for breast cancer prognosis. He has acted as consultant for Roche, Eisai, Celgene, Pierre-Fabre, and Sividon and has received honoraria from AstraZeneca, Novartis, Pfizer, Essex, Celgene, Pierre-Fabre, GlaxoSmithKline, Lilly, Sanofi-Aventis, Roche, Sividon, Genomic Health, and Amgen. He has received previous funding from Siemens Healthcare Diagnostics Products GmbH, Sanofi-Aventis, and the Bundesministerium für Bildung und Forschung (BMBF).

\section{References}

-1 Peto R, Davies C, Godwin J, Gray R, Pan HC, et al.: Comparisons between different polychemotherapy regimens for early breast cancer: metaanalyses of long-term outcome among 100,000 women in 123 randomised trials. Lancet 2012; 379:432-444.

2 Goldhirsch A, Winer EP, Coates AS, Gelber RD, Piccart-Gebhart M, et al.: Personalizing the treatment of women with early breast cancer: highlights of the St Gallen International Expert Consensus on the Primary Therapy of Early Breast Cancer 2013. Ann Oncol 2013;24:2206-2223.

3 Simon RM, Paik S, Hayes DF: Use of archived specimens in evaluation of prognostic and predictive biomarkers. J Natl Cancer Inst 2009;101:14461452.

4 Harris L, Fritsche H, Mennel R, Norton L, Ravdin P, et al.: American Society of Clinical Oncology 2007 update of recommendations for the use of tumor markers in breast cancer. J Clin Oncol 2007;25: 5287-5312.

5 Paik S, Shak S, Tang G, Kim C, Baker J, et al.: A multigene assay to predict recurrence of tamoxifen-treated, node-negative breast cancer. N Engl J Med 2004;351:2817-2826.

6 Paik S, Tang G, Shak S, Kim C, Baker J, et al.: Gene expression and benefit of chemotherapy in women with node-negative, estrogen receptorpositive breast cancer. J Clin Oncol 2006;24:37263734.

7 Albain KS, Barlow WE, Shak S, Hortobagyi GN, Livingston RB, et al.: Prognostic and predictive value of the 21-gene recurrence score assay in postmenopausal women with node-positive, oestrogenreceptor-positive breast cancer on chemotherapy: a retrospective analysis of a randomised trial. Lancet Oncol 2010;11:55-65.

8 Filipits M, Rudas M, Jakesz R, Dubsky P, Fitzal F, et al.: A new molecular predictor of distant recur- rence in ER-positive, HER2-negative breast cancer adds independent information to conventional clinical risk factors. Clin Cancer Res 2011;17:60126020.

9 Dubsky P, Filipits M, Jakesz R, Rudas M, Singer CF, et al.: EndoPredict improves the prognostic classification derived from common clinical guidelines in ER-positive, HER2-negative early breast cancer. Ann Oncol 2013;24:640-647.

$\checkmark 10$ van 't Veer LJ, Dai H, van de Vijver MJ, He YD, Hart AAM, et al.: Gene expression profiling predicts clinical outcome of breast cancer. Nature 2002;415:530-536.

11 Perou CM, Sørlie T, Eisen MB, van de Rijn M, Jeffrey SS, et al.: Molecular portraits of human breast tumours. Nature 2000;406:747-752.

12 Parker JS, Mullins M, Cheang MCU, Leung S, Voduc D, et al.: Supervised risk predictor of breast cancer based on intrinsic subtypes. J Clin Oncol 2009;27:1160-1167.

13 Cortazar P, Zhang L, Untch M, Mehta K, Costantino JP, et al.: Pathological complete response and long-term clinical benefit in breast cancer: the CTNeoBC pooled analysis. Lancet 2014, doi: 10.1016/S0140-6736(13)62422-8 [Epub ahead of print].

14 Kaufmann M, von Minckwitz G, Mamounas EP, Cameron D, Carey LA, et al.: Recommendations from an international consensus conference on the current status and future of neoadjuvant systemic therapy in primary breast cancer. Ann Surg Oncol 2012;19:1508-1516.

15 von Minckwitz G, Blohmer JU, Costa SD, Denkert C, Eidtmann H, et al.: Response-guided neoadjuvant chemotherapy for breast cancer. J Clin Oncol 2013;31:3623-3630.

16 von Minckwitz G, Schneeweiss A, Loibl S, Salat C, Denkert C, et al.: Neoadjuvant carboplatin in patients with triple-negative and HER2-positive early breast cancer (GeparSixto; GBG 66): a randomised phase 2 trial. Lancet Oncol. 2014, doi: 10.1016/ S1470-2045(14)70160-3 [Epub ahead of print].

17 Bonadonna G, Brusamolino E, Valagussa P, Rossi A, Brugnatelli L, et al.: Combination chemotherapy as an adjuvant treatment in operable breast cancer. N Engl J Med 1976;294:405-410.

18 Levine MN, Pritchard KI, Bramwell VHC, Shepherd LE, Tu D, et al.: Randomized trial comparing cyclophosphamide, epirubicin, and fluorouracil with cyclophosphamide, methotrexate, and fluorouracil in premenopausal women with nodepositive breast cancer: update of National Cancer Institute of Canada Clinical Trials Group Trial MA5. J Clin Oncol 2005;23:5166-5170.

19 Henderson IC, Berry DA, Demetri GD, Cirrincione CT, Goldstein LJ, et al.: Improved outcomes from adding sequential paclitaxel but not from escalating doxorubicin dose in an adjuvant chemotherapy regimen for patients with node-positive primary breast cancer. J Clin Oncol 2003;21:976983.

20 Roché H, Fumoleau P, Spielmann M, Canon J, Delozier T, et al.: Sequential adjuvant epirubicinbased and docetaxel chemotherapy for node-positive breast cancer patients: the FNCLCC PACS 01 Trial. J Clin Oncol 2006;24:5664-5671.

21 Martín M, Lluch A, Seguí MA, Ruiz A, Ramos M, et al.: Toxicity and health-related quality of life in breast cancer patients receiving adjuvant docetaxel, doxorubicin, cyclophosphamide (TAC) or 5-fluorouracil, doxorubicin and cyclophosphamide (FAC): impact of adding primary prophylactic granulocyte-colony stimulating factor to the TAC regimen. Ann Oncol 2006;17:1205-1212.

22 Mackey JR, Martin M, Pienkowski T, Rolski J, Guastalla J, et al.: Adjuvant docetaxel, doxorubicin, and cyclophosphamide in node-positive breast cancer: 10-year follow-up of the phase 3 ran- 
domised BCIRG 001 trial. Lancet Oncol 2013; 14:72-80.

23 Schmidt M, Victor A, Bratzel D, Boehm D, Cotarelo C, et al.: Long-term outcome prediction by clinicopathological risk classification algorithms in node-negative breast cancer - comparison between Adjuvant!, St Gallen, and a novel risk algorithm used in the prospective randomized NodeNegative-Breast Cancer-3 (NNBC-3) trial. Ann Oncol 2009;20:258-264.

24 Martín M, Seguí MA, Antón A, Ruiz A, Ramos M, et al.: Adjuvant docetaxel for high-risk, nodenegative breast cancer. N Engl J Med 2010;363: 2200-2210.

-25 Eiermann W, Pienkowski T, Crown J, Sadeghi S, Martin M, et al.: Phase III study of doxorubicin/ cyclophosphamide with concomitant versus sequential docetaxel as adjuvant treatment in patients with human epidermal growth factor receptor 2-normal, node-positive breast cancer: BCIRG-005 trial. J Clin Oncol 2011;29:3877-3884.

-26 Citron ML, Berry DA, Cirrincione C, Hudis C, Winer EP, et al.: Randomized trial of dose-dense versus conventionally scheduled and sequential versus concurrent combination chemotherapy as postoperative adjuvant treatment of node-positive primary breast cancer: first report of Intergroup Trial C9741/Cancer and Leukemia Group B Trial 9741. J Clin Oncol 2003;21:1431-1439.

27 Moebus V, Jackisch C, Lueck H, Du Bois A, Thomssen $\mathrm{C}$, et al.: Intense dose-dense sequential chemotherapy with epirubicin, paclitaxel, and cyclophosphamide compared with conventionally scheduled chemotherapy in high-risk primary breast cancer: mature results of an AGO phase III study. J Clin Oncol 2010;28:2874-2880.

28 Bonilla L, Ben-Aharon I, Vidal L, Gafter-Gvili A, Leibovici L, et al.: Dose-dense chemotherapy in nonmetastatic breast cancer: a systematic review and meta-analysis of randomized controlled trials. J Natl Cancer Inst 2010;102:1845-1854.

29 Sparano JA, Wang M, Martino S, Jones V, Perez EA, et al.: Weekly paclitaxel in the adjuvant treatment of breast cancer. N Engl J Med 2008; 358:1663-1671.
30 Swain SM, Tang G, Geyer CE, Rastogi P, Atkins JN, et al.: Definitive results of a phase III adjuvant trial comparing three chemotherapy regimens in women with operable, node-positive breast cancer: the NSABP B-38 trial. J Clin Oncol 2013;31:31973204.

31 Shulman LN, Cirrincione CT, Berry DA, Becker HP, Perez EA, et al.: Six cycles of doxorubicin and cyclophosphamide or paclitaxel are not superior to four cycles as adjuvant chemotherapy for breast cancer in women with zero to three positive axillary nodes: Cancer and Leukemia Group B 40101. J Clin Oncol 2012;30:4071-4076.

32 Jones S, Holmes FA, O'Shaughnessy J, Blum JL, Vukelja SJ, et al.: Docetaxel with cyclophosphamide is associated with an overall survival benefit compared with doxorubicin and cyclophosphamide: 7-year follow-up of US Oncology Research Trial 9735. J Clin Oncol 2009;27:1177-1183.

33 Giordano SH, Lin Y, Kuo YF, Hortobagyi GN, Goodwin JS: Decline in the use of anthracyclines for breast cancer. J Clin Oncol 2012;30:2232-2239. 